\title{
AVALIAÇÃO DE SIMULADORES APLICADOS À SÍNTESE DE TECIDOS junto a alunos de Medicina Veterinária
}

\author{
Comparison of simulators for tissue suture training by \\ Veterinary Medicine students
}

\author{
Aline Machado De Zoppa ${ }^{1}$, Andrea Barbosa ${ }^{1}$, Greyce Lousana ${ }^{2}$, Thaís Sodré2*(1) \\ *Autor Correspondente: Thaís Sodré. Rua Itaipu, 475, Mirandópolis, \\ São Paulo, SP, CEP: 04052-010 \\ E-mail: thais@invitare.com.br
}

\begin{abstract}
Como citar: ZOPPA, Aline Machado De et al. Avaliação de Simuladores Aplicados à Síntese de Tecidos junto a alunos de Medicina Veterinária. Revista de Educação Continuada em Medicina Veterinária e Zootecnia do CRMV-SP, São Paulo, v.19, n.1, 2021, e38097. Doi 10.36440/recmvz.v19i1.38097
\end{abstract}

Cite as: ZOPPA, Aline Machado De et al. Comparison of simulators for tissue suture training by Veterinary Medicine students. Journal of Continuing Education in Veterinary Medicine and Animal Science of CRMV-SP, São Paulo, v.19, n.1, 2021, e38097. Doi 10.36440/recmvz.v19i1.38097

\section{Resumo}

O presente estudo avaliou a efetividade de simulador de sutura de silicone frente ao modelo usual em feltro e bastidor no desenvolvimento da habilidade em síntese cirúrgica. Vinte e seis alunos de Medicina Veterinária, sem vivência cirúrgica, foram distribuídos em dois grupos: o primeiro utilizou modelo de simulador em silicone e o segundo o simulador em bastidor e feltro. Em três encontros foram abordados conceitos sobre síntese, tipos de sutura e treinamento prático. Para avaliar sua experiência e percepções, os alunos responderam a um questionário e foram avaliados pelos professores na confecção dos padrões de sutura. Na avaliação do questionário, o simulador em silicone foi superior em sete das 10 questões. Na avaliação dos docentes, não houve diferença entre os grupos. Concluímos que ambos os modelos foram efetivos no desenvolvimento da habilidade cirúrgica, porém, a aceitabilidade dos alunos foi superior no modelo inovador.

Palavras-chave: Simulador. Técnica Cirúrgica. Silicone. Sutura.

\section{Abstract}

This study compared the efficiency of a novel silicone model to a traditional low-fidelity method on the acquisition of surgical synthesis skills. Twenty-six veterinary students with no prior surgical training were randomly allocated into two groups: the first used a novel silicon model and second trained on a traditional set up. During three sessions, subjects such as synthesis, suture patterns, and suture practice were discussed. In order to assess the students experiences and perceptions, a questionary was applied. Students were also evaluated by teachers who scored their suture patterns. The questionary showed that the silicone model was superior in 7 out

\footnotetext{
1 Docente do curso de Medicina Veterinária, Universidade Anhembi Morumbi, São Paulo, SP, Brasil

2 Médica-veterinária, Invitare Pesquisa Clínica, São Paulo, SP, Brasil
} 
of 10 questions. No significant differences were detected between groups on the assessment by the teachers. This study shows that either model is effective for the development of suture skills. Nonetheless, the novel silicone model tested here has better acceptability when compared with the traditional model.

Keywords: Simulator. Surgical Technique. Silicone. Suture.

\section{Introdução}

A formação cirúrgica envolve complexos conceitos teóricos e práticos, particularmente relacionados à técnica operatória e suas fases fundamentais, para os quais o desenvolvimento de habilidades e destreza são considerados essenciais (COSTA NETO et al., 2010).

Neste âmbito, a síntese cirúrgica é uma das etapas mais importantes da cirurgia, uma vez que favorece a efetiva restituição dos tecidos incisados. Sua prática, além de conhecimento, requer habilidades psicomotoras. A falta de agilidade e destreza do cirurgião, nesse sentido, são prejudiciais para a reconstituição tecidual e, consequentemente, constituem fator adicional de risco em procedimentos cirúrgicos (MARTINS FILHO, 2015). O treinamento, a partir da instrução e repetição, determina o maior impacto na qualidade do aprendizado (BLAU et al., 2020), que reflete no maior interesse e competência dos alunos (BASTOS; SILVA, 2011). Com isso, há interesse crescente nas instituições de ensino de Medicina e Medicina Veterinária pela implementação de laboratórios especializados no desenvolvimento de habilidades cirúrgicas.

Nesse moderno conceito de educação, as habilidades cirúrgicas básicas são ensinadas e praticadas em simuladores, considerados seguros, reprodutíveis e portáteis (DENADAI; OSHIIWA; SAADHOSSNE, 2012; REZNICK; MACRAE, 2006; WANZEL; WARD; REZNICK, 2002). Outro fator importante a ser considerado na presente demanda por modelos simuladores é a crescente consciência da sociedade em relação ao bem-estar animal, principalmente no que concerne à utilização de animais vivos para práticas iniciais de ensino (BASTOS e SILVA, 2011), custo elevado e necessidade de estrutura e profissionais especializados (WANZEL; WARD; REZNICK, 2002). Também tem sido relatada a maior tranquilidade dos alunos em desenvolver determinada habilidade inicialmente em um simulador, para posteriormente aplicá-la com maior confiança em um paciente real (BASTOS e SILVA, 2011; DENADAI; OSHIIWA; SAAD-HOSSNE, 2012). Outros destacam a melhora significativa no escore de parâmetros avaliados após o treinamento, independente do material utilizado, reforçando assim o potencial do simulador quando comparado ao tecido original (BLAU et al., 2020; DENADAI, OSHIIWA, SAAD-HOSSNE, 2012; WANZEL; WARD; REZNICK, 2002). Por essa razão, tais métodos já não são mais considerados por muitos educadores como alternativos e sim como parte essencial do processo de aprendizagem, sendo comprovadamente eficazes (GROBER et al., 2004).

Nesse contexto, a utilização de método de baixo custo elaborado com tecido preso a bastidor para o aprendizado da síntese e dos respectivos padrões de sutura é amplamente difundida no Brasil, mesmo considerando o baixo potencial realístico desse simulador. Neto et al. (2012) quantificaram os índices de aceitação desse método em acadêmicos da disciplina de Técnica Cirúrgica e relataram excelente grau de aceitação e incremento na sedimentação do conhecimento. Entre as vantagens desse método sobre o treinamento com língua bovina destacam-se a durabilidade do simulador e a facilidade de transporte, permitindo o treinamento de padrões de suturas por repetidas vezes sem a necessidade de ambiente apropriado para sua utilização.

Já estão disponíveis no mercado internacional, inclusive para a área de Medicina Veterinária, modelos de sutura que oferecem experiências ainda mais realísticas. Tais modelos em silicone mimetizam as diferentes camadas teciduais, incluindo derme, tecido subcutâneo, gordura e musculatura. No entanto, o elevado custo de importação desses produtos inviabiliza a difusão do seu uso no mercado nacional.

Diante do exposto, o objetivo do presente estudo prospectivo de não inferioridade foi avaliar a efetividade de simulador de sutura de silicone produzido no Brasil no desenvolvimento da habilidade 
em síntese cirúrgica em alunos voluntários do curso de Medicina Veterinária, sem vivência cirúrgica prévia, frente ao modelo usual utilizado, composto por estrutura de tecido e bastidor.

\section{Material e métodos}

O estudo foi conduzido a partir da avaliação comparativa do desempenho dos alunos de Medicina Veterinária após treinamento de técnica de síntese cirúrgica em modelo convencional (tecido e bastidor) e em simulador de silicone.

\section{Análise ética do projeto}

O protocolo do presente estudo, inserido na Plataforma Brasil, por possuir participantes voluntários humanos, foi avaliado e aprovado pelo Comitê de Ética em Pesquisa da Universidade Anhembi Morumbi sob o protocolo número 3.634.894.

\section{Recrutamento dos participantes}

O projeto foi divulgado para alunos cursando o sétimo semestre do curso de Medicina Veterinária, sem experiência prévia com técnicas de sutura. Os 26 alunos que participaram do projeto preencheram e assinaram um Termo de Consentimento Livre e Esclarecido que foi entregue à equipe do estudo antes do início das atividades práticas.

\section{Materiais}

Foram avaliados dois modelos de simuladores de sutura. Um deles, utilizado atualmente na disciplina de Técnica Cirúrgica da instituição, constituído por um bastidor de $30 \mathrm{~cm}$ de diâmetro no qual foi fixado feltro. 0 segundo simulador foi um modelo fabricado à base de silicone, contendo três camadas distintas (representando pele, subcutâneo e musculatura), com $21 \times 15 \mathrm{~cm}$ de largura e 0,8 $\mathrm{cm}$ de altura.

0 instrumental cirúrgico utilizado fazia parte do acervo da instituição. No primeiro encontro foi utilizado fio de nylon pesca $0,35 \mathrm{~mm}$, acoplado em agulha $3 / 8$ trifacetada de $2 \mathrm{~cm}$, porém nos treinamentos subsequentes, o fio foi substituído pelo de nylon preto agulhado (Brasture).

\section{Treinamento}

O presente estudo compreendeu cinco encontros presenciais de duas horas cada. No primeiro, o delineamento do estudo foi apresentado aos participantes e o Termo de Consentimento Livre e Esclarecido foi entregue, preenchido e assinado pelos alunos.

No segundo encontro, foi realizada a distribuição randomizada dos 26 alunos entre os grupos simulador feltro no bastidor (GB) e simulador em silicone (GS) e se deu início à apresentação dos conceitos de síntese cirúrgica e à atividade prática. Os dois encontros seguintes foram dedicados para aprimoramento da técnica de sutura dos pontos simples interrompidos, caminhando para pontos Sultan, Colchoeiro, simples contínuo, U contínuo, Lembert e Cushing (Figura 1A e 1B). 
Figura 1: Fotografias dos simuladores após treinamento de alunos do curso de Medicina Veterinária na realização de técnica de sutura cirúrgica. A: simulador em silicone; B: simulador feltro em bastidor.

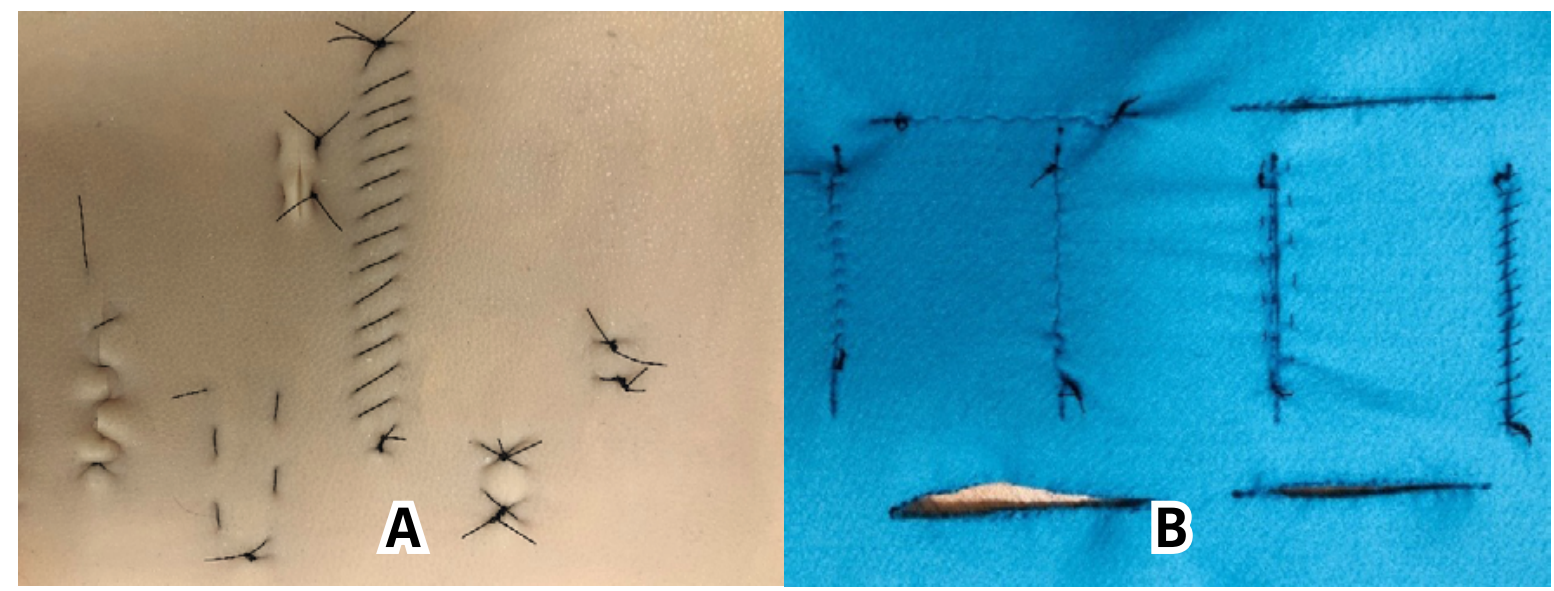

T Fonte: Os autores (2019).

No quinto e último encontro participaram apenas os alunos que cumpriram a carga horária anterior. Os alunos responderam a um questionário de avaliação pós-treinamento e foram submetidos à avaliação prática realizada por docentes da disciplina de Técnica Cirúrgica no mesmo modelo de simulador em que o treinamento foi realizado.

\section{Questionário pós-treinamento}

Após a conclusão da etapa prática, os alunos de ambos os grupos responderam um questionário com 10 questões de Análises psicométricas adaptado de Martins Filho (2015), destinado a avaliar a experiência e as percepções do aluno frente aos simuladores utilizados. Os itens poderiam ser respondidos em cinco questões de múltipla escolha em escala de Likert (discordo completamente; discordo; não concordo nem discordo; concordo e concordo completamente).

\section{Avaliação prática}

A avaliação prática individual dos alunos foi realizada por docentes da disciplina de Técnica Cirúrgica da instituição. Os alunos realizaram os diferentes tipos de suturas apresentados durante o treinamento com o respectivo simulador utilizado durante essa fase. Foram avaliados os parâmetros gerais relacionados à correta aproximação das bordas da ferida, realização adequada do nó e do ponto cirúrgico, finalização da sutura, posicionamento das mãos durante a confecção do nó e no manejo do instrumental, perfazendo um total de 10 itens. 0 docente classificou cada um dos itens avaliados em realização total, parcial, ou não realização.

\section{Análise estatística}

Para a avaliação estatística do questionário, uma média (nota) sobre o uso do simulador foi construída a partir da média aritmética dos 10 itens reescalonados para variar entre 0 e 100, onde 10 são percepções mais positivas sobre o simulador. Para esse cálculo, considerou-se que todos os itens estavam em escala crescente, onde discordar totalmente era uma resposta negativa e concordar totalmente uma resposta positiva ao item.

As distribuições por item e da média foram então comparadas entre tipo do simulador (Silicone e Bastidor/feltro) utilizando teste não paramétrico de Mann-Whitney (HOLLANDER e WOLFE, 1999) com nível de significância de 5\% (Figura 2). 
Figura 2: Boxplot representando a média, desvio padrão e distribuição das respostas de questionário de análises psicométricas dos alunos do curso de Medicina Veterinária que receberam treinamento na realização da técnica de sutura cirúrgica com dois tipos de simuladores.

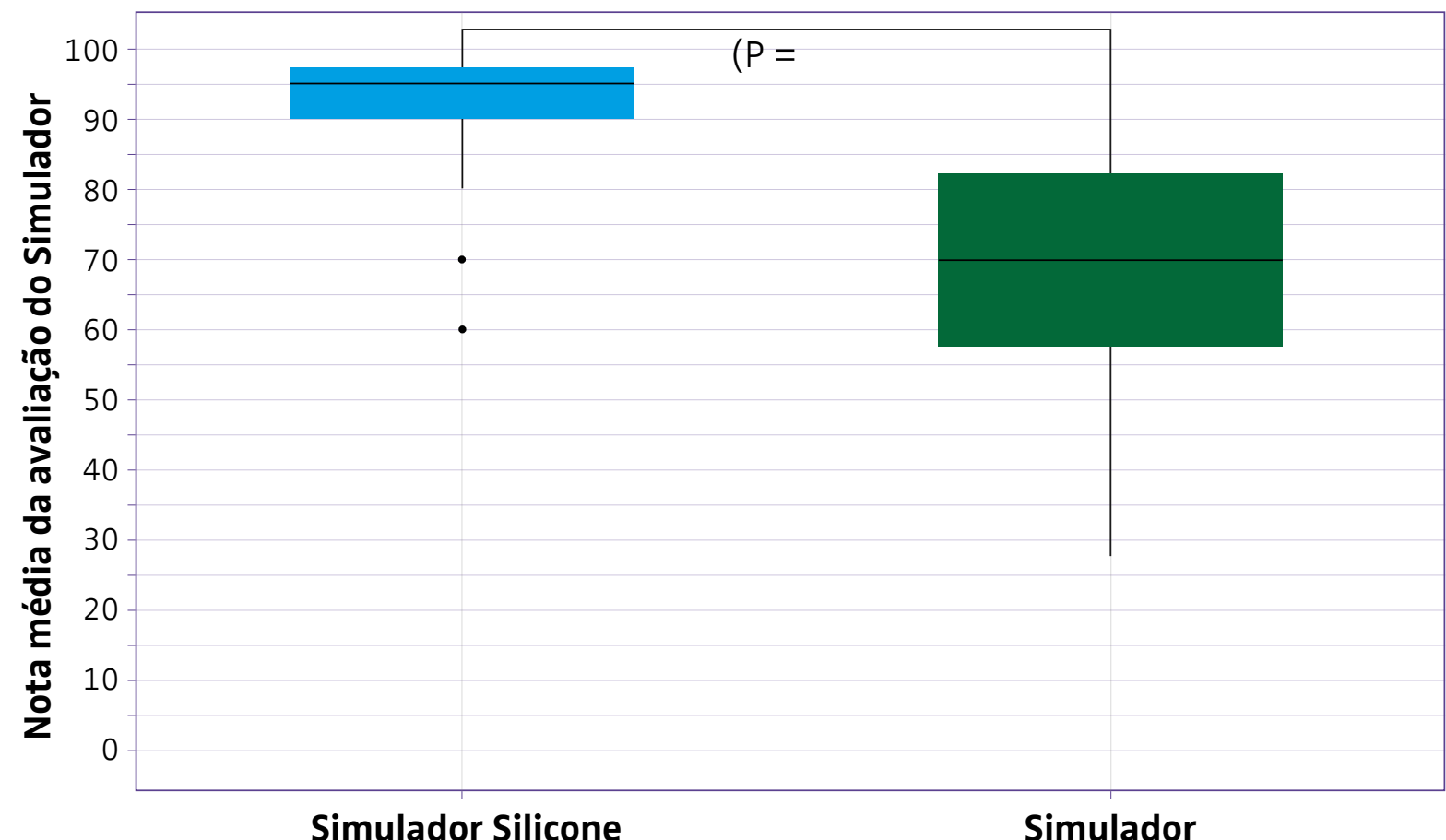

TFonte: Os autores (2019).

Os professores, por sua vez, avaliaram os alunos em 10 procedimentos indicando três respostas possíveis: realização total, parcial, ou não realização. A nota final do docente foi calculada de forma similar ao questionário dos alunos e comparada por teste de Mann-Whitney, já seus itens, um a um, foram avaliados por teste exatos de Fisher (AGRESTI, 2002).

As análises foram realizadas com auxílio do software R 3.6.1 (R Core Team, 2019).

\section{Resultados}

\section{Questionário pós-treinamento}

Os resultados das respostas do questionário foram apresentados em frequências absolutas e relativas para cada item do questionário. Ao nível de significância de $5 \%$, sete das dez questões apresentaram resultados mais favoráveis para utilização do simulador em silicone (Tabela 1), sendo observado valor de $p \leq 0,001$ em quatro questões e de $p \leq 0,05$ em outras três. Não foi observada diferença entre os grupos nas questões que abordaram as dimensões e peso do simulador frente a facilidade de manuseio do simulador, a experiência por não utilizar animais vivos no desenvolvimento de habilidades básicas para síntese dos tecidos e a necessidade em desenvolver novos métodos inovadores de ensino que não utilizem animais vivos, particularmente na técnica cirúrgica. 
Tabela 1: Percentual de respostas obtidas no questionário de análises psicométricas aplicado após treinamento de alunos, em curso de graduação de Medicina Veterinária, com o emprego de dois tipos de simuladores para treinamento na realização de suturas.

\begin{tabular}{|c|c|c|c|c|c|c|}
\hline & \multirow{2}{*}{\multicolumn{5}{|c|}{ RESPOSTAS }} \\
\hline & & & & & & \\
\hline Perguntas & Grupo & $\begin{array}{l}\text { Discordo } \\
\text { comple- } \\
\text { tamente }\end{array}$ & Discordo & $\begin{array}{c}\text { Não con- } \\
\text { cordo nem } \\
\text { discordo }\end{array}$ & Concordo & $\begin{array}{l}\text { Concordo } \\
\text { completa- } \\
\text { mente }\end{array}$ \\
\hline \multirow{2}{*}{$\begin{array}{l}\text { 1. O simulador utilizado é apropriado } \\
\text { para o desenvolvimento de habilidades } \\
\text { relativas à síntese de tecidos? }\end{array}$} & GS & - & - & - & $15 \%$ & $85 \%$ * \\
\hline & GB & - & $15 \%$ & $8 \%$ & $62 \%$ & $15 \%$ \\
\hline \multirow{2}{*}{$\begin{array}{l}\text { 2. O simulador utilizado estimula o } \\
\text { treinamento de padrões de sutura? }\end{array}$} & GS & - & - & $8 \%$ & $8 \%$ & $85 \%+$ \\
\hline & GB & $8 \%$ & $15 \%$ & $8 \%$ & $23 \%$ & $46 \%$ \\
\hline \multirow{2}{*}{$\begin{array}{l}\text { 3. As dimensões e o peso do simulador } \\
\text { facilitam seu manuseio? }\end{array}$} & GS & - & $8 \%$ & $8 \%$ & $54 \%$ & $31 \%$ \\
\hline & GB & $15 \%$ & $23 \%$ & $15 \%$ & $15 \%$ & $31 \%$ \\
\hline \multirow{2}{*}{$\begin{array}{l}\text { 4. Esse simulador propiciou uma } \\
\text { experiência agradável por não utilizar } \\
\text { animais vivos no desenvolvimento } \\
\text { de habilidades básicas para síntese } \\
\text { dos tecidos? }\end{array}$} & GS & - & - & $8 \%$ & $15 \%$ & $77 \%$ \\
\hline & $\mathrm{GB}$ & - & - & $8 \%$ & $38 \%$ & $54 \%$ \\
\hline \multirow{2}{*}{$\begin{array}{l}\text { 5. Os conceitos relacionados ao padrão } \\
\text { de sutura podem ser aplicados com } \\
\text { esse simulador? }\end{array}$} & GS & - & - & - & $8 \%$ & $92 \%$ * \\
\hline & $\mathrm{GB}$ & - & - & - & $77 \%$ & $23 \%$ \\
\hline \multirow{2}{*}{$\begin{array}{l}\text { 6. } 0 \text { treinamento relacionado à síntese } \\
\text { dos tecidos proporcionado por esse } \\
\text { simulador permiteo desenvolvimento de } \\
\text { outras habilidades cirúrgicas, como, por } \\
\text { exemplo, manipulação do instrumental? }\end{array}$} & GS & - & - & - & $23 \%$ & $77 \%+$ \\
\hline & $\mathrm{GB}$ & - & $8 \%$ & $15 \%$ & $38 \%$ & $38 \%$ \\
\hline \multirow{2}{*}{$\begin{array}{l}\text { 7. A integridade dos materiais que } \\
\text { compõem o simulador é mantida com } \\
\text { a repetição das atividades de síntese? }\end{array}$} & GS & - & $23 \%$ & $23 \%$ & $46 \%$ & $8 \%$ * \\
\hline & $\mathrm{GB}$ & $31 \%$ & $62 \%$ & $8 \%$ & - & - \\
\hline \multirow{2}{*}{$\begin{array}{l}\text { 8. } 0 \text { simulador utilizado facilitou o } \\
\text { aprendizado das técnicas relativas à } \\
\text { síntese de suturas? }\end{array}$} & GS & - & - & - & $31 \%$ & $69 \%+$ \\
\hline & GB & - & $8 \%$ & $8 \%$ & $69 \%$ & $15 \%$ \\
\hline \multirow{2}{*}{$\begin{array}{l}\text { 9. Você recomendaria esse simulador } \\
\text { para o ensino de habilidades relativas } \\
\text { à síntese de tecidos? }\end{array}$} & GS & - & - & - & $15 \%$ & $85 \%$ * \\
\hline & $\mathrm{GB}$ & - & $31 \%$ & $31 \%$ & $23 \%$ & $15 \%$ \\
\hline \multirow{2}{*}{$\begin{array}{l}\text { 10. Na sua opinião, existe a necessi- } \\
\text { dade de desenvolver novos métodos } \\
\text { inovadores de ensino que não utilizem } \\
\text { animais vivos, particularmente na téc- } \\
\text { nica cirúrgica? }\end{array}$} & GS & - & - & - & $23 \%$ & $77 \%$ \\
\hline & GB & - & - & $8 \%$ & $15 \%$ & $77 \%$ \\
\hline
\end{tabular}

Valor $p$ teste de Mann-whitney: ${ }^{\star}: p \leq 0,001 ;+: p \leq 0,05$. GS = grupo simulador em silicone; GB: grupo simulador de feltro em bastidor.

TFonte: Os autores (2019). 
A nota média final dos questionários apontou percepções mais positivas para o grupo que utilizou o simulador em silicone $(89,4 \pm 12,1)$ quando comparado ao grupo que utilizou Feltro em Bastidor $(68,1 \pm 20,4)$, com $p=0,003$.

\section{Avaliação prática}

0 resultado da avaliação prática realizada pelos docentes em cada um dos grupos é apresentado na Tabela 2. Assim como para a avaliação do questionário, criou-se uma nota final do docente, baseada na soma dos 10 itens. A nota final variou de 0 a 100 pontos, assumindo que realizar o procedimento valia 10 pontos, realizar parcialmente 5 pontos e não realizar, 0 pontos.

Tabela 2: Percentual de conceitos obtidos na avaliação pós-treinamento.

\begin{tabular}{|c|c|c|c|c|}
\hline & \multicolumn{3}{|c|}{ RESPOSTAS } \\
\hline Perguntas & Grupo & Realizou & $\begin{array}{c}\text { Realizou } \\
\text { parcialmente }\end{array}$ & $\begin{array}{c}\text { Não } \\
\text { realizou }\end{array}$ \\
\hline \multirow{2}{*}{$\begin{array}{l}\text { 1. Realizou correta aproximação das } \\
\text { bordas da ferida? }\end{array}$} & GS & $100 \%$ & - & - \\
\hline & GB & $85 \%$ & $15 \%$ & - \\
\hline \multirow{2}{*}{$\begin{array}{l}\text { 2. A sutura foi realizada sem sobrepo- } \\
\text { sição e com espaço entre os pontos de } \\
0,5 \mathrm{~cm} \text { ? }\end{array}$} & GS & $92 \%$ & $8 \%$ & - \\
\hline & $\mathrm{GB}$ & $85 \%$ & $8 \%$ & $8 \%$ \\
\hline \multirow{2}{*}{$\begin{array}{l}\text { 3. Foi realizado nó não apertado e ao } \\
\text { lado da incisão? }\end{array}$} & GS & $85 \%$ & $15 \%$ & - \\
\hline & $\mathrm{GB}$ & $62 \%$ & $38 \%$ & - \\
\hline \multirow{2}{*}{$\begin{array}{l}\text { 4. O tipo de ponto foi confeccionado } \\
\text { adequadamente? }\end{array}$} & GS & $92 \%$ & $8 \%$ & - \\
\hline & $\mathrm{GB}$ & $100 \%$ & - & - \\
\hline \multirow{2}{*}{$\begin{array}{l}\text { 5. O nó final foi realizado correta- } \\
\text { mente em suturas contínuas? }\end{array}$} & GS & $100 \%$ & - & - \\
\hline & $\mathrm{GB}$ & $100 \%$ & - & - \\
\hline \multirow{2}{*}{$\begin{array}{l}\text { 6. A sutura realizada chegou até o final } \\
\text { da linha de incisão? }\end{array}$} & GS & $100 \%$ & - & - \\
\hline & $\mathrm{GB}$ & $100 \%$ & - & - \\
\hline \multirow{2}{*}{ 7. As bordas ficaram bem coaptadas? } & GS & $100 \%$ & - & - \\
\hline & $\mathrm{GB}$ & $100 \%$ & - & - \\
\hline \multirow{2}{*}{$\begin{array}{l}\text { 8. Os instrumentos foram portados da } \\
\text { maneira correta? }\end{array}$} & GS & $85 \%$ & - & $15 \%$ \\
\hline & GB & $100 \%$ & - & - \\
\hline \multirow{2}{*}{$\begin{array}{l}\text { 9. } 0 \text { porta-agulhas foi posicionado cor- } \\
\text { retamente? }\end{array}$} & GS & $46 \%$ & $8 \%$ & $46 \%$ \\
\hline & $\mathrm{GB}$ & $62 \%$ & $15 \%$ & $23 \%$ \\
\hline \multirow{2}{*}{ 10. A tesoura foi utilizada corretamente? } & GS & $46 \%$ & $38 \%$ & $15 \%$ \\
\hline & GB & $77 \%$ & $23 \%$ & - \\
\hline
\end{tabular}

GS: Grupo Simulador em Silicone; GB: Grupo Feltro em Bastidor.

T Fonte: Os autores (2019). 
Não foi observada diferença significativa entre os grupos em nenhum dos itens avaliados, relacionados à adequação das suturas, o tipo de ponto realizado, à finalização da sutura, bem como ao posicionamento das mãos na confecção dos nós ou no manejo do instrumental. Da mesma forma, não foi observada diferença na nota final alcançada pelo grupo simulador em silicone $(88,1 \pm 15,2)$ quando comparada ao grupo simulador feltro em bastidor $(91,9 \pm 12,3)$.

\section{Discussão}

0 presente estudo foi delineado para avaliar a aceitabilidade e funcionalidade de simulador em silicone comparado ao modelo amplamente utilizado nas aulas de técnica cirúrgica em nosso país (simulador de feltro em bastidor). Uma das propostas de avaliação seguiu a aplicação de questionário assim como realizado por Martins Filho (2015). Nesse contexto, o simulador em silicone apresentou avaliação geral superior quando comparado ao modelo em feltro e bastidor, com ênfase nos quesitos relacionados à adequação para o desenvolvimento das habilidades de síntese em tecidos, estímulo ao treinamento, possibilidade de aplicação dos conceitos de sutura, desenvolvimento de outras habilidades tais como manipulação do instrumental e facilidade de aprendizado. Ainda $100 \%$ dos alunos avaliados responderam que recomendariam a utilização do simulador em silicone para 0 ensino de habilidades relativas às sínteses dos tecidos. No grupo feltro em bastidor somente $38 \%$ dos alunos fariam essa recomendação.

Uma vez que se sabe que a partir do treinamento repetido se obtém melhor resultado no aprendizado de uma técnica, a durabilidade e portabilidade do simulador devem ser consideradas. Neto et al. (2012) relataram excelente grau de aceitação e incremento na sedimentação do conhecimento com a utilização do método do tecido em bastidor, no entanto, nosso estudo mostrou que embora não tenha sido observada diferença entre os simuladores no que tange às dimensões e peso, 93\% dos alunos que utilizaram o simulador de feltro em bastidor apresentaram uma percepção negativa em relação à manutenção da integridade do material com o treinamento repetido. No grupo que utilizou o simulador em silicone, 54\% apresentaram percepção positiva e apenas 23\% relataram percepção negativa.

Bastos e Silva (2011) e Blau et al. (2020) consideram o estímulo para treinamento regular importante no engajamento do aluno e na determinação de um melhor aprendizado. De fato, no presente trabalho, foi constatado que $23 \%$ dos alunos que utilizaram o simulador de feltro em bastidor relataram percepções negativas em relação ao estímulo para o treino de padrões de sutura enquanto nenhum dos alunos do grupo simulador em silicone relatou percepções negativas. De forma contrária, o simulador em silicone serviu como estímulo ao treinamento para 93\% dos alunos. Essa melhor percepção entre os alunos na utilização de um modelo mais realista quando comparado ao treinamento utilizando-se outros materiais também confirma os resultados referidos por Wanzel; Ward; Reznick (2002) e Williamson et al. (2017). Acreditamos que nesse quesito, mais uma vez a manutenção de integridade do dispositivo ao longo de sua utilização deve refletir de forma positiva no estímulo ao treinamento continuado.

Todos os alunos do grupo simulador em silicone consideraram que o dispositivo utilizado facilitou o aprendizado e responderam positivamente quanto ao desenvolvimento de habilidades relativas à síntese de tecidos, aplicação de conceitos de padrões de sutura, além do desenvolvimento de outras habilidades cirúrgicas, como por exemplo a manipulação de instrumental. Ainda que seja descrita na literatura uma boa aceitabilidade dos métodos de simulador de sutura de baixo custo (BASTOS e SILVA, 2011; COSTA NETO et al., 2012), verificamos que o simulador em silicone despertou percepções mais positivas. Isso mostra que o modelo mais realista parece despertar mais interesse e consequentemente facilitar o treinamento, permitindo um melhor desenvolvimento de habilidades relacionadas à técnica cirúrgica e vai ao encontro da avaliação realizada em alunos de Medicina Veterinária por Williamson et al. (2017), onde o aumento na fidelidade do simulador de sutura abdominal promoveu incremento significativo na habilidade adquirida dos alunos. 
Ainda sobre o questionário, observamos comportamento semelhante entre os grupos quando o tema foi a não utilização de animais vivos para o treinamento e a necessidade de desenvolvimento de métodos inovadores indo ao encontro da tendência de desenvolvimento de uma educação humanitária, que não faz uso de animais vivos (BACHINSKI et al., 2017).

Desta maneira, os simuladores seguros, reprodutíveis e portáteis que foram descritos como ideais por autores como Wanzel et al. (2002), Reznick e MacRae (2006) e Denadai; Oshiiwa; SaadHossne (2012) podem ser considerados de grande importância para o ensino atual da técnica cirúrgica. Essa tecnologia facilita e permite o treinamento das principais técnicas aplicadas, dispensando a utilização de animais vivos ou até mesmo de materiais biológicos, que podem ser diretamente afetadas pela crescente consciência pelo bem-estar animal, descrita por Bastos e Silva (2011); Wanzel; Ward; Reznick (2002); e Bachinski et al. (2017).

No quesito da avaliação prática, os presentes resultados também confirmam as descrições da literatura de Blau et al. (2020), Wanzel et al. (2002) e Denadai; Oshiiwa; Saad-Hossne (2012), destacando a importância da instrução e treinamento para o aprendizado e desenvolvimento de uma boa técnica de sutura, independente do material utilizado. Adicionalmente, os resultados demonstram a não inferioridade do modelo inovador em silicone frente ao modelo consagrado.

\section{Conclusão}

0 presente estudo nos permite concluir que os dois simuladores utilizados para o ensino de técnicas de sutura se mostraram eficientes quanto ao aproveitamento do ensino, independentemente do modelo utilizado. No entanto, a avaliação do questionário confirmou a preferência dos alunos pela utilização do modelo em silicone que, por ser mais realístico, pode trazer outros benefícios relacionados à prática da sutura cirúrgica contribuindo positivamente para a qualidade do aprendizado.\&

\section{Referências}

AGRESTI, A. Categorical data analysis. 2. ed. New York: Wiley, 2002, 734 p.

BACHINSKI, R. et al. On the journey toward humane education in Brazil: first request for a total ban of harmful animal use in professional and higher education. Alternatives to laboratory animals, ATLA, v.45, n. 5, p. 287-293, nov. 2017. DOl: https://doi.org/10.1177/026119291704500501.

BASTOS, E. M.; SILVA, R. D. P. Proposal of a synthetic ethylene-vinyl acetate bench model for surgical foundations learning: suture training. Acta Cirúrgica Brasileira, v.26, n. 2, p. 149-152, abr. 2011. DOl: https://doi.org/10.1590/S0102-86502011000200014.

BLAU, J. A. et al. Does Realism Matter? A randomized controlled trial comparing models for medical student suture education. Plastic and Reconstructive Surgery Global Open, v.8, n. 4, e2738, apr. 2020. DOI: https://doi.org/10.1097/GOX.0000000000002738.

COSTA NETO, J. M.; MARTINS FILHO, E. F. Sistemas educacionais hipermídia como ferramenta de apoio ao ensino da Medicina Veterinária. Ciência Veterinária nos Trópicos, v. 13, n. 1, p. 126-130, 2010. Suplemento.

COSTA NETO, J. M. et al. Bastidor aplicado ao ensino da técnica cirúrgica veterinária - síntese dos tecidos. Medvep - Revista Científica de Medicina Veterinária - Pequenos Animais e Animais de Estimação, $\mathrm{n}$. 10, v. 32, p. 1-6, 2012.

DENADAI, R.; OSHIIWA, M.; SAAD-HOSSNE, R. Does bench model fidelity interfere in the acquisition of suture skills by novice medical students. Revista da Associação Médica Brasileira, v. 58, n. 5, p. 600-606, 2012. DOI: https://doi.org/10.1590/S0104-42302012000500019. 
GROBER, E. D. et al. The educational impact of bench model fidelity on the acquisition of technical skill: the use of clinically relevant outcome measures. Annals of Surgery, v. 240, n. 2, p. 374-381, aug. 2004. DOl: https://doi.org/10.1097/01.sla.0000133346.07434.30.

HOLLANDER, M.; WOLFE, D.A. Nonparametric Statistical Methods. 2. ed. New York: John Wiley \& Sons, 1999, $816 \mathrm{p}$.

MARTINS FILHO, E.F. Métodos alternativos no ensino da técnica cirúrgica veterinária. 2015. Tese (Doutorado em Cirurgia veterinária) - Faculdade de Ciências Agrárias e Veterinárias, Universidade Estadual de São Paulo, Campus de Jaboticabal, Jaboticabal, 2015. 101p.

REZNICK, R. K.; MACRAE, H. Teaching surgical skills - Changes in the wind. The New England Journal of Medicine, v.355, n. 25, p. 2664-2669, dec. 2006. DOl: https://doi.org/10.1056/NEJMra054785.

WILLIAMSON, J. et al. The impact of model fidelity on acquisition of three-layer abdominal incision closure skills in novice veterinary students. In: UNIVERSITY OF PRETORIA, Faculty of Veterinary Science. Proceedings of the 5th International Veterinay Simulation in Teaching Conference. Pretoria: Faculty of Veterinary Science, University of Pretoria, 2017.

WANZEL, K. R.; WARD, M.; REZNICK, R. K. Teaching the surgical craft: from selection to certification. Current Problems in Surgery, v.39, n. 6, p. 583-659, jun. 2002. DOI: https://doi.org/10.1067/mog.2002.123481. 